\title{
Editorial
}

\section{International Conference on Acoustics and Vibration 2012}

\author{
Hamid Mehdigholi, ${ }^{1,2}$ Hamid Ahmadian, ${ }^{3}$ and Abdolreza Ohadi ${ }^{4}$ \\ ${ }^{1}$ International Communication Committee of ISAV, Iran \\ ${ }^{2}$ Department of Mechanical Engineering, Sharif University of Technology, Tehran 14588-89695, Iran \\ ${ }^{3}$ School of Mechanical Engineering, Iran University of Science and Technology, Narmak, Tehran 16846, Iran \\ ${ }^{4}$ Acoustics Research Laboratory, Department of Mechanical Engineering, Amirkabir University of Technology, Hafez Avenue 424, \\ Tehran 15916-34311, Iran
}

Correspondence should be addressed to Abdolreza Ohadi; a_r_ohadi@aut.ac.ir

Received 19 November 2012; Accepted 22 November 2012; Published 10 July 2014

Copyright ( $) 2014$ Hamid Mehdigholi et al. This is an open access article distributed under the Creative Commons Attribution License, which permits unrestricted use, distribution, and reproduction in any medium, provided the original work is properly cited.

The Second International Conference on Acoustics and Vibration (ISAV2012) was held on December 26-27, 2012, at the Sharif University of Technology, Tehran, Iran. This is an annual conference organized by the Iranian Society of Acoustics and Vibration (ISAV) in order that the researchers and engineers share their findings and mutual concerns in this field.

The growing significance of acoustics and vibration in all branches of engineering is fully clear, which undoubtedly has led to new grounds for improving and serving the society. Vibrations, more precisely, mechanical vibrations, as the main field along with acoustics-as they complement one another-express their vitality in all aspects of engineering.

Among the 450 abstracts that were received for the conference, 280 were selected for a review process. During the review process by three experts for each paper, 243 papers were chosen for presentation at the conference. 44 papers were included in a poster session; the remaining papers were presented in 8 parallel sessions in two days. The conference Scientific Committee nominated the top research papers to be published in the special issues of a well-known journal. The committee asked the authors of the 11 selected English papers to declare their agreement and also make further improvements to their papers for Shock and Vibration journal, resulting in this special issue.

\section{Acknowledgment}

On behalf of the organizing committee of the conference, I would like to express my sincere thanks to the researchers who contributed to the success of the conference by presenting their research findings at the conference. In particular, I would like to express my appreciation to the authors of the 11 papers selected for this special issue for their archival contribution.

Hamid Mehdigholi Hamid Ahmadian Abdolreza Ohadi 

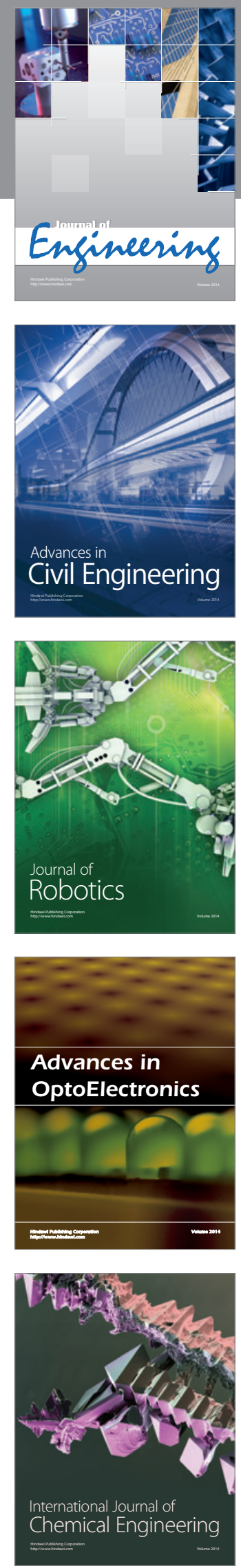

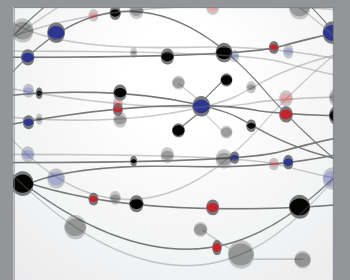

The Scientific World Journal
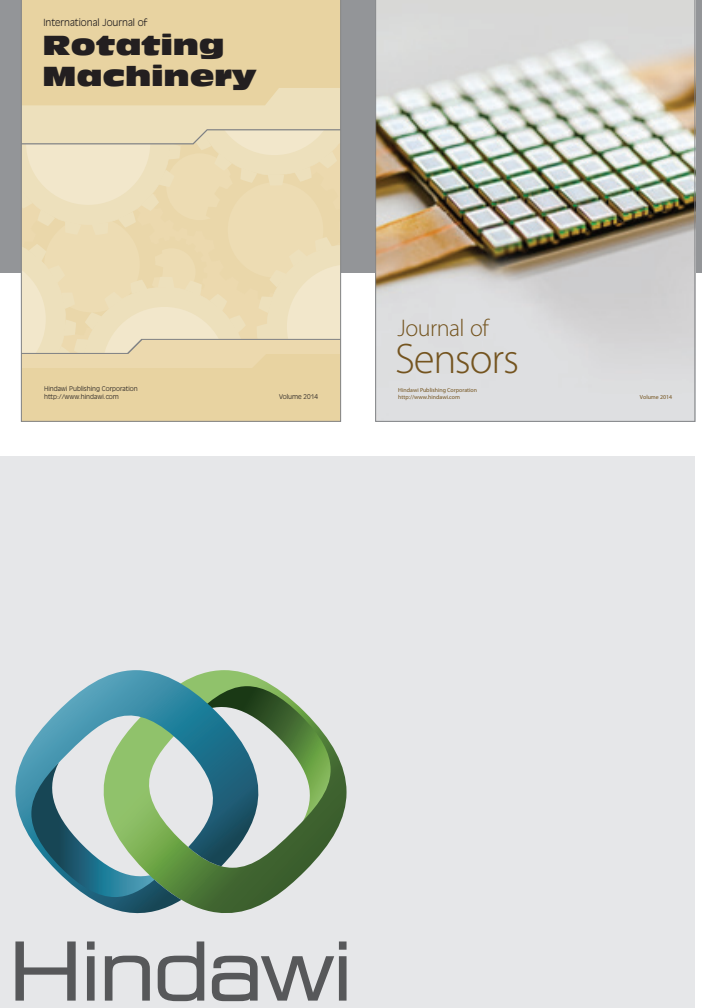

Submit your manuscripts at http://www.hindawi.com
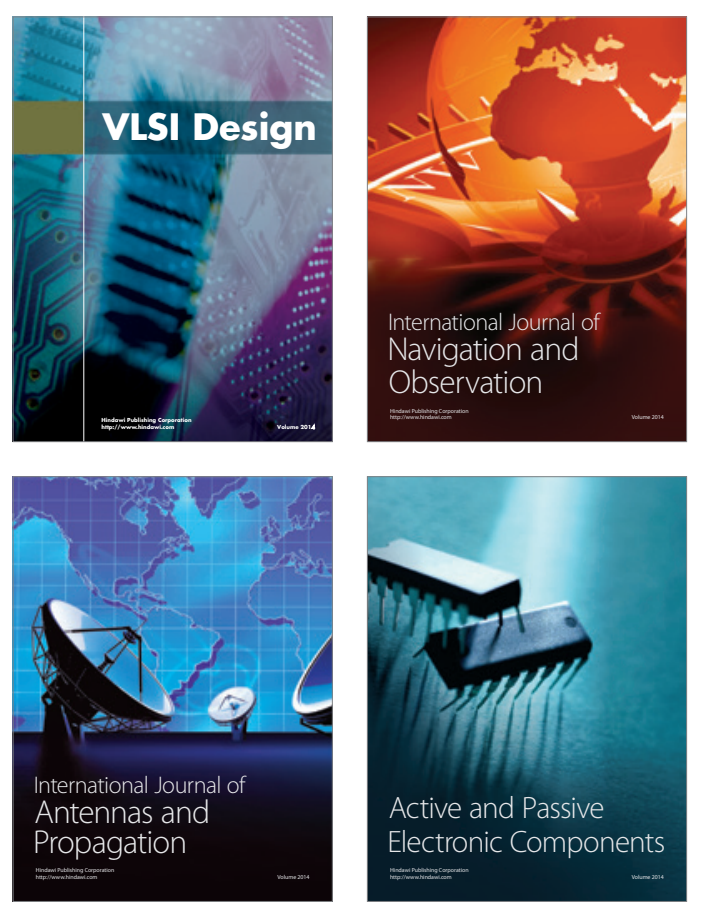
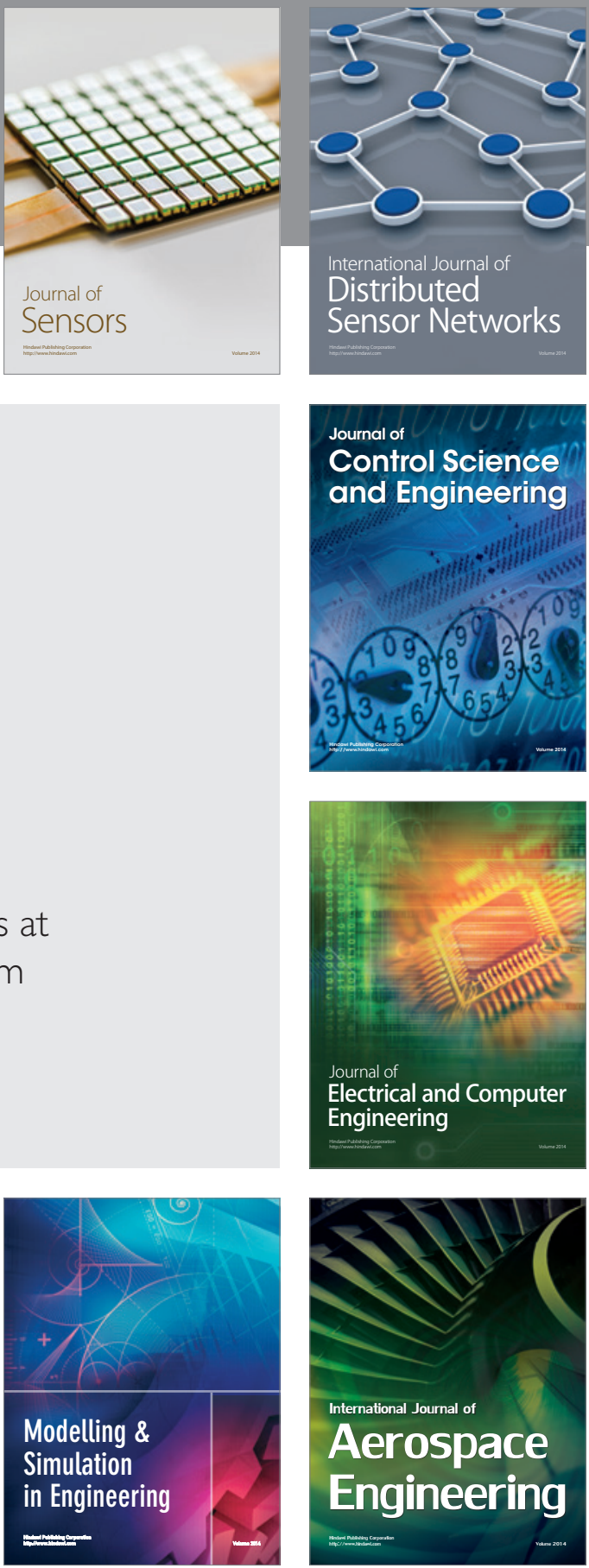

Journal of

Control Science

and Engineering
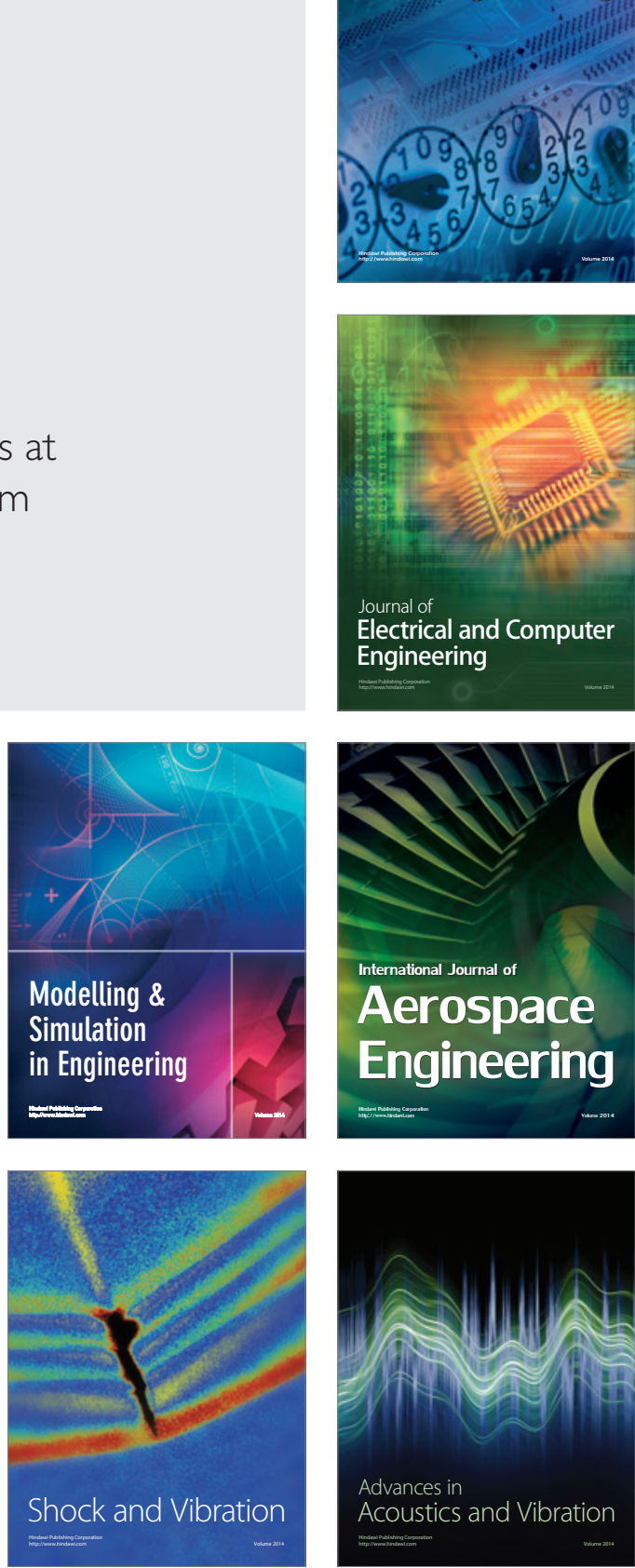